\title{
Levantamento de limites de imóvel rural a partir de fotografias obtidas por VANT, apoiadas por pontos GNSS
}

\author{
Gilda Maria Dias* \\ Jeanine Cassini Peter* \\ Marcelo Pereira* \\ Ronaldo dos Santos da Rocha* \\ Mário Reiss*
}

Recebido el 27 de abril de 2015; aceito el 26 de junho de 2015

\section{Resumen}

Con la popularización de los VANT (Vehículos Aéreos No Tripulados), la aerofotogrametría para el mapeamiento de pequeñas áreas, está convirtiendo una alternativa cada vez más viable, proporcionando resolución espacial adecuada para su finalidad. En este trabajo, la georeferenciación de inmuebles rurales, basado en el recubrimiento aerofotogramétrico con VANT, fue desarrollado en un área de aproximadamente 80ha. Esta propuesta de levantamiento indirecto de divisas naturales en regiones de difícil acceso, es presentada como alternativa al levantamiento geodésico con receptores GNSS, y así atender los requisitos de precisión posicional establecidos en el Manual Técnico de Posicionamiento del INCRA (Instituto Nacional de Colonizaciones y Reforma Agraria). En la etapa de ejecución, se realizaron levantamientos con el uso de receptores GNSS y levantamiento fotogramétrico con un VANT de pequeño porte para el mapeamiento planimétrico. Como resultado del proceso fotogramétrico, fue generada una ortofoto y una carta planimétrica resultante de la vectorización de características sobre ortofoto. Para el control de calidad, fueron comparadas coordenadas de puntos en la ortofoto y en la carta con coordenadas de puntos homólogos en el terreno, llegando a una exactitud de $0.279 \mathrm{~m}$ para $90 \%$ de las coordenadas extraídas

* Departamento de Geodésia, Universidade Federal do Rio Grande do Sul (UfrgS), Av. Bento Gonçalves, 9500, CEP 91501-970, Porto Alegre - RS, Brasil, e-mail: gildinha.dias@gmail.com, jepeter@gmail.com, marcelo.cartografo@gmail.com, ronaldo.rocha@ufrgs.br, mario.reiss@ufrgs.br 
de la ortofoto y $0.281 \mathrm{~m}$ para las coordenadas resultantes del mapa. Estos valores son compatibles con la Norma Técnica del INCRA, que exige precisión de $3.0 \mathrm{~m}$ para límites naturales y de $7.5 \mathrm{~m}$ para límites inaccesibles. El control de calidad fue basado en el padrón de exactitud cartográfica para cartografía digital (PEC-PCD) y a través de este control se verificó que el producto fuera catalogado como Clase A para la escala 1:1,000.

Palabras clave: georeferenciación, VANT, registro rural.

\section{Abstract}

With the popularity of UAVs (Unmanned Aerial Vehicles), the aerial photography methaod for mapping small areas has become an increasingly viable alternative, providing spatial resolution adequate to its end. In this study, the georeferencing of rural properties, based on aerial photographs covering using UAV, it wasdeveloped in an area of approximately 80 ha. Was developed as a proposition of indirect measurement of natural boundaries in areas of difficult access and as an alternative to geodetic survey with GNSS receivers, in order to comply with accuracy requirements set out in INCRA's Technical Manual of Positioning. In the implementation stage, data collection was made with use of GNSS receivers and photogrammetric survey with a small UAV for planimetric mapping. As a result of the photogrammetric process, an orthophoto and a planimetric map resulting of the vectoring of the ortophoto ware generated. For quality control, a comparison was made with coordinates of the points in the orthophoto and the map with the coordinates of homologous points on the ground, coming to an accuracy of 0.279 $\mathrm{m}$ for $90 \%$ of the extracted coordinates of the orthophoto and $0.281 \mathrm{~m}$ for the resulting coordinates of the map. These values are compatible to INCRA's Technical Standard, which requires precision of $3.0 \mathrm{~m}$ for natural limits and $7.5 \mathrm{~m}$ for inaccessible limits. Quality control was based on Cartographic Accuracy Standard for Digital Cartography (PEC-PCD) and, through statistical test, it was found that the product can be classified in Class A in scale 1:1,000.

Key words: geocoding, UAV, rural registration.

\section{Resumo}

Com a popularização dos VANT (Veículos Aéreos Não Tripulados), a aerofotogrametria para mapeamento de pequenas áreas tem se tornado uma alternativa cada vez mais viável, proporcionando resolução espacial adequada ao seu fim. Neste trabalho, o georreferenciamento de imóveis rurais, baseado no recobrimento aerofotogramétrico com VANT, foi desenvolvido em uma área de aproximadamente 80 ha. Esta proposta de levantamento indireto de divisas naturais em regiões de difícil acesso é apresentada como alternativa ao levantamento 
geodésico com receptores GNSS, de forma a atender requisitos de precisão posicional estabelecidos no Manual Técnico de Posicionamento do INCRA - Instituto Nacional de Colonização e Reforma Agrária. Na etapa de execução, foram realizados levantamentos com uso de receptores GNSS e levantamento fotogramétrico com um VANT de pequeno porte para o mapeamento planimétrico. Como resultado do processo fotogramétrico, foi gerada uma ortofoto e uma carta planimétrica resultante da vetorização de feições sobre a ortofoto. Para o controle de qualidade, foram comparadas coordenadas de pontos na ortofoto e na carta com coordenadas de pontos homólogos no terreno, chegando-se a uma acurácia de 0,279 $\mathrm{m}$ para $90 \%$ das coordenadas extraídas da ortofoto e $0,281 \mathrm{~m}$ para as coordenadas resultantes da carta. Esses valores são compatíveis com a Norma Técnica do INCRA, que exige precisão de $3,0 \mathrm{~m}$ para limites naturais e de 7,5 m para limites inacessíveis. $\mathrm{O}$ controle de qualidade baseou-se no Padrão de Exatidão Cartográfica para a Cartografia Digital (PEC-PCD) e, através de teste estatístico, verificou-se que o produto pode ser classificado como Classe A para a escala 1:1.000.

Palavras chave: georreferenciamento, VANT, cadastro rural.

\section{Introdução}

Grande parte do território brasileiro é ocupada por propriedades rurais. Em 2000, $81,25 \%$ dos brasileiros que residiam em áreas urbanas ocupavam apenas $1,1 \%$ do território (Instituto Brasileiro de Geografía e Estatística - IBGE, 2014). Grande parte das propriedades rurais não está mapeada ou regularizada em um sistema efetivo de controle territorial. Leis e decretos foram estabelecidos nos últimos anos na tentativa de organizar um sistema eficaz para constituir um apoio a ações administrativas, públicas e de planejamento.

A promulgação da Lei 10.267, de 28 de agosto de 2001, conhecida como a Lei do Georreferenciamento, regulamentou a criação de um sistema de cadastro para regularizar a situação dos imóveis rurais no país. O Cadastro Nacional de Imóveis Rurais (CNIR) é o novo sistema de cadastro de terras que cria uma base de dados única e comum entre o Instituto Nacional de Colonização e Reforma Agrária (INCRA), os Registros de Imóveis, a Receita Federal e outras instituições. Os limites e confrontações do imóvel rural ou urbano de uso agrícola passaram a ser caracterizados pelas coordenadas dos vértices que compõem o perímetro, georreferenciadas ao Sistema Geodésico Brasileiro. Antes da Lei, não havia padrão para o registro da geometria e características do imóvel.

Sucessivos atos normativos foram homologados desde então para orientar os serviços de georreferenciamento de imóveis rurais. Técnicas de sensoriamento remoto são permitidas com restrições por estas normas. As precisões para definição dos limites de propriedades foram definidas pela Norma Técnica de Georreferenciamento de Imóveis Rurais. As precisões requeridas para limites 
inacessíveis oferecem maior tolerância do que para limites secos. Prazos para regularização de todos os imóveis rurais do país também foram estabelecidos. Com o objetivo de realizar o georreferenciamento de uma área rural, o trabalho foi realizado observando as precisões estabelecidas e aplicabilidade da fotogrametria aérea com uso de Veículo Aéreo Não Tripulado (VANT). O desenvolvimento do trabalho é composto de levantamentos geodésicos e fotogramétricos em uma área de estudo. A análise da qualidade geométrica dos dados obtidos também se insere nos objetivos propostos para análise posicional planimétrica.

\section{Fundamentação teórica}

\section{Aspectos legais e normativos}

Algumas leis e normas vigoraram no país com o objetivo de regulamentar o cadastro rural, iniciando-se pela Lei $\mathrm{n}^{\circ} 4.504$, de 30 de novembro de 1964, cujos objetivos cercavam o aspecto fiscal, análises microeconômicas, planos assistenciais, colonização e regularização de posseiros. A promulgação da Lei $\mathrm{n}^{\circ} 10.267$ e suas regulamentações alteraram leis anteriores e criou um novo cadastro de imóveis rurais, denominado CNIR (Cadastro Nacional de Imóveis Rurais). Este cadastro constitui-se em uma base comum de dados a ser gerenciada pelo INCRA e a Secretaria da Receita Federal a ser produzida e compartilhada por instituições públicas federais e estaduais produtoras e usuárias de informações sobre o meio rural brasileiro. A descrição dos imóveis passou a ser caracterizada pelas coordenadas dos vértices que compõem o perímetro, georreferenciadas ao Sistema Geodésico Brasileiro, com precisão posicional a ser fixada pelo INCRA. O memorial descritivo deve ser assinado por um profissional habilitado, cabendo ao referido Instituto certificar que as poligonais dos imóveis não se sobreponham (Brasil, 2001).

As atuais normativas para execução dos serviços de agrimensura para fins de certificação, foram homologadas através da Portaria INCRA $\mathrm{n}^{0} 486$, de 02 de setembro de 2013 e compreendem a Norma Técnica para Georreferenciamento de Imóveis Rurais (NTGIR), 3a. edição, o Manual Técnico de Posicionamento e o Manual Técnico de Limites e Confrontações. A NTGIR estabelece valores de precisão posicional de $0,50 \mathrm{~m}$, ou melhor, para limites artificiais, $3,00 \mathrm{~m}$ para limites naturais e $7,50 \mathrm{~m}$ para limites inacessíveis. O Manual Técnico de Posicionamento define que o levantamento dos vértices do imóvel pode ser realizado por métodos como posicionamento por GNSS e topografia convencional e restringe o uso de métodos de sensoriamento remoto para levantamento de vértices em divisas naturais ou inacessíveis. Estabelece também a utilização do Sistema Geodésico Local (SGL) para o cálculo de área (INCRA, 2013). O Manual Técnico de Limites e Confrontações orienta a identificação e descrição dos limites dos imóveis 
e os casos de alteração de parcelas certificadas e determina a guarda e manutenção, pelo responsável, de todo o material que subsidiou o levantamento, para dirimir possíveis dúvidas ou divergências futuras quanto à localização de limites (INCRA, 2013).

\section{Posicionamento por satélites artificiais}

Neste trabalho utilizou-se o posicionamento por Global Navigation Satellite System (GNSS) em várias etapas, para georreferenciar as informações espaciais ao Sistema Geodésico Brasileiro (SGB). Foram utilizados os posicionamentos relativo estático e RTK (Real Time Kinematic). A precisão alcançada no uso do posicionamento relativo é influenciada por vários fatores, dentre eles o comprimento das linhas base e o tempo de rastreio. Segundo Prado e Krueger (2003), para linhas de base com comprimento inferior a $1,5 \mathrm{~km}$, o erro planimétrico será menor que $3 \mathrm{~cm}$ e para linhas de base, variando de 1,5 a $25 \mathrm{~km}$, esse erro é de 3 a $7 \mathrm{~cm}$.

O IBGE apresenta as precisões aproximadas do posicionamento relativo (Tabela 1), informando que são valores de referência. Outros fatores também interferem na qualidade dos rastreios, e também devem ser observados.

Tabela 1

Precisões aproximadas obtidas com o posicionamento relativo por GNSS

\begin{tabular}{lcll}
\hline Linha de Base & Tempo de Observação & Equipamento & \multicolumn{1}{c}{ Precisão } \\
\hline $00-05 \mathrm{~km}$ & $05-10 \mathrm{~min}$ & $\mathrm{~L} 1 \mathrm{ou} \mathrm{L} 1 / \mathrm{L} 2$ & $5-10 \mathrm{~mm}+1 \mathrm{ppm}$ \\
$05-10 \mathrm{~km}$ & $10-15 \mathrm{~min}$ & $\mathrm{~L} 1 \mathrm{ou} \mathrm{L} 1 / \mathrm{L} 2$ & $5-10 \mathrm{~mm}+1 \mathrm{ppm}$ \\
$10-20 \mathrm{~km}$ & $10-30 \mathrm{~min}$ & $\mathrm{~L} 1 \mathrm{ou} \mathrm{L} 1 / \mathrm{L} 2$ & $5-10 \mathrm{~mm}+1 \mathrm{ppm}$ \\
$20-50 \mathrm{~km}$ & $02-03 \mathrm{~h}$ & $\mathrm{~L} 1 / \mathrm{L} 2$ & $5 \mathrm{~mm}+1 \mathrm{ppm}$ \\
$50-100 \mathrm{~km}$ & Mínimo $03 \mathrm{~h}$ & $\mathrm{~L} 1 / \mathrm{L} 2$ & $5 \mathrm{~mm}+1 \mathrm{ppm}$ \\
$>100 \mathrm{~km}$ & Mínimo $04 \mathrm{~h}$ & L1/L2 & $5 \mathrm{~mm}+1 \mathrm{ppm}$ \\
\hline
\end{tabular}

Fonte: IBGE, 2008.

\section{Qualidade cartográfica}

A qualidade posicional de um documento cartográfico pode ser avaliada segundo dois critérios: precisão e acurácia. A primeira está vinculada a efeitos apenas aleatórios (à dispersão das observações), enquanto que acurácia vincula-se a erros aleatórios e sistemáticos. A acurácia posicional indica a qualidade da posição planialtimétrica de uma grandeza medida ou parâmetro estimado, quando comparada com uma grandeza de qualidade posicional superior. De acordo com 
Merchant (1982, apud Oliveira, 2011), dados utilizados como referência posicional de documentos cartográficos devem ter uma acurácia pelo menos três vezes maior que a do produto analisado.

Conforme o Decreto $\mathrm{n}^{\mathrm{o}}$ 89.817, de 20 de junho de 1984, que estabelece as Instruções Reguladoras das Normas Técnicas da Cartografia Nacional, a classificação de uma carta através de valores de Padrão de Exatidão Cartográfica (PEC) e o Erro Padrão (EP) para planimetria e altimetria é dada pela Tabela 2, onde Ec significa escala da carta e Eq, equidistância.

Tabela 2

Classificação da carta segundo o PEC, de 1984

\begin{tabular}{ccccc}
\hline \multirow{2}{*}{ Classe } & \multicolumn{2}{c}{ Planimetria $(*)$} & \multicolumn{2}{c}{ Altimetria(**) } \\
& $P E C(\mathrm{~mm})$ & $E P(\mathrm{~mm})$ & $P E C(\mathrm{~mm})$ & $E P(\mathrm{~mm})$ \\
\hline $\mathrm{A}$ & $0,5 \times \mathrm{Ec}$ & $0,3 \times \mathrm{Ec}$ & $1 / 2 \times \mathrm{Eq}$ & $1 / 3 \times \mathrm{xq}$ \\
$\mathrm{B}$ & $0,8 \times \mathrm{Ec}$ & $0,5 \times \mathrm{Ec}$ & $3 / 5 \times \mathrm{Eq}$ & $2 / 5 \times \mathrm{Eq}$ \\
$\mathrm{C}$ & $1,0 \times \mathrm{Ec}$ & $0,6 \times \mathrm{Ec}$ & $3 / 4 \times \mathrm{Eq}$ & $1 / 2 \times \mathrm{Eq}$ \\
\hline
\end{tabular}

Fonte: Brasil, 1984.

Tabela 3

Classificação da carta conforme proposto através do PEC-PCD

\begin{tabular}{ccccccccc}
\hline \multirow{2}{*}{$P E C-$} & \multicolumn{2}{c}{$1: 1.000$} & \multicolumn{2}{c}{$1: 2.000$} & \multicolumn{2}{c}{$1: 5.000$} & \multicolumn{2}{c}{$1: 10.000$} \\
\cline { 2 - 9 } PCD & $P E C$ & $E P$ & $P E C$ & $\begin{array}{c}E P \\
(m)\end{array}$ & $\begin{array}{c}P E C \\
(m)\end{array}$ & $\begin{array}{c}E P \\
(m)\end{array}$ & $\begin{array}{c}P E C \\
(m)\end{array}$ & $\begin{array}{c}E P \\
(m)\end{array}$ \\
\hline A & 0,28 & 0,17 & 0,56 & 0,34 & 1,40 & 0,85 & 2,80 & 1,70 \\
B & 0,50 & 0,30 & 1,00 & 0,60 & 2,50 & 1,50 & 5,00 & 3,00 \\
C & 0,80 & 0,50 & 1,60 & 1,00 & 4,00 & 2,50 & 8,00 & 5,00 \\
D & 1,00 & 0,60 & 2,00 & 1,20 & 5,00 & 3,00 & 10,00 & 6,00 \\
\hline
\end{tabular}

Fonte: Adaptado de CONCAR-EB, 2011.

Segundo Rocha (2002), observa-se que o avanço da informática e os recursos tecnológicos utilizados na elaboração de uma carta resultaram em ganho de qualidade na aquisição das informações, no processamento, no gerenciamento de dados e na forma de representação. Assim, o chamado PEC Digital (PEC-PCD) é uma proposição da Comissão Nacional de Cartografia (CONCAR) em substituição ao PEC. Os valores propostos são apresentados na Tabela 3. 


\section{Análise da qualidade geométrica}

$\mathrm{Na}$ análise da qualidade geométrica, são comparadas as coordenadas do ponto obtidas no mapa com as coordenadas homólogas levantadas em campo, obtidas por técnica de elevada precisão (Rocha, 2002). Na avaliação de mapas digitais, cujo parâmetro considerado é a exatidão cartográfica, é necessário definir o tamanho amostral, os tipos de amostras e os métodos de análise que validarão a qualidade. Numa ortofoto digital, o número populacional pode ser estimado em proporção a quantidade de informações mapeadas ou pela razão entre o tamanho da área mapeada e o tamanho do pixel no terreno. Conforme Rocha, "são exigidos vinte ou mais pontos teste requeridos para conduzir a uma significante avaliação estatística da exatidão [...]". Para o Padrão Nacional para Exatidão de Dados Espaciais (NSSDA) dos EUA, 20 pontos proporcionam um processamento ao nível de confiança de $95 \%$ e, em sendo menos que isso, não pode ser aplicado (Rocha, 2002).

\section{Análise estatística de tendência e de precisão}

Galo e Camargo (1994), baseados em Merchant (1982), apresentaram um método para complementar a legislação e analisar a consistência dos dados. O método consiste na análise estatística das discrepâncias entre as componentes $\mathrm{E}$ e $\mathrm{N}$ das coordenadas dos pontos de controle, através das análises de tendência e de precisão. A análise de tendência baseia-se no teste de hipótese, onde se verifica se a média das discrepâncias $(\overline{\Delta X})$ é estatisticamente igual ou diferente de zero com base na distribuição $t$ de Student (Lima et al., 2014). O valor da estatística amostral $t$ é calculado pela equação (1):

$$
t_{x=\left(\frac{\Delta X}{S_{\Delta X}}\right) * \sqrt{n}}
$$

Onde $S_{\Delta X}$ é o desvio-padrão das discrepâncias.

De acordo com Lima et al. (2014):

O intervalo de confiança é definido por $|\mathrm{tx}|<\mathrm{t} \alpha / 2, \mathrm{n}-1$ e, uma vez que a estatística amostral $t$ não satisfaz essa desigualdade, a ortofoto não pode ser considerada como livre de tendências significativas na coordenada testada para um determinado nível de significância $(\alpha)$.

A análise de precisão é feita comparando-se o desvio-padrão das discrepâncias com o EP especificado para uma determinada classe. Verifica-se, através de um teste de hipótese, se a variância das discrepâncias (S2 $\Delta \mathrm{X})$ é estatisticamente igual ou maior que a variância especificada, de acordo com o EP $(\sigma x)$, baseando-se na distribuição Qui-quadrado. 
O valor da estatística amostral $\chi^{2}$ é calculado pela equação (2).

$$
\chi_{x}^{2}=(n-1)\left(\frac{s_{\Delta X}^{2}}{\sigma_{X}^{2}}\right)
$$

Ainda conforme Lima et al. (2014), "levando-se em conta que o EP é fixado para a resultante, pois o desvio-padrão é o mesmo para as coordenadas $\mathrm{E}$ e $\mathrm{N}$, a variância para uma determinada classe poder ser calculada pela equação" (3).

$$
\sigma_{X}^{2}=\left(\frac{E P^{2}}{2}\right)
$$

O intervalo de confiança é definido por $\chi_{x}^{2} \leq \chi_{\alpha, n-1}^{2}$. Se a estatística amostral Qui-quadrado não satisfizer essa desigualdade, o produto cartográfico não atende a precisão pré-estabelecida (Lima et al., 2014).

\section{Metodologia}

\section{Área de estudo}

Localiza-se no município de Eldorado do Sul, cuja posição geográfica é $30^{\circ} 05^{\prime}$ 27" S e 51 40' 18" W, no Estado do Rio Grande do Sul (Figura 1). A área de estudo é parte do Assentamento São Pedro, do INCRA, e possui aproximadamente 87 ha. O Assentamento teve seu início em 1986 e sua demarcação topográfica foi realizada, primeiramente, em 1995, por topografia convencional. Em 2011, o Instituto fez um novo levantamento usando receptores GNSS com o propósito de atender aos requisitos de precisão posicional estabelecidos na Norma Técnica do INCRA.

\section{Levantamentos geodésicos}

A aquisição dos dados foi realizada em três etapas. Na primeira, levantamento geodésico com receptores GNSS, foram levantados marcos da rede geodésica local e o levantamento das coordenadas de feições na área selecionada. Na segunda etapa, foram feitas a pré-sinalização dos pontos de apoio ao levantamento aerofotogramétrico com a aquisição de coordenadas nos centros geométricos dos alvos, por GNSS, seguida da realização do voo. A terceira etapa foi realizada para aquisição de pontos de checagem necessários ao controle da qualidade geométrica dos produtos gerados. A Figura 3 apresenta a pré-sinalização dos pontos de apoio ao levantamento aerofotogramétrico. 


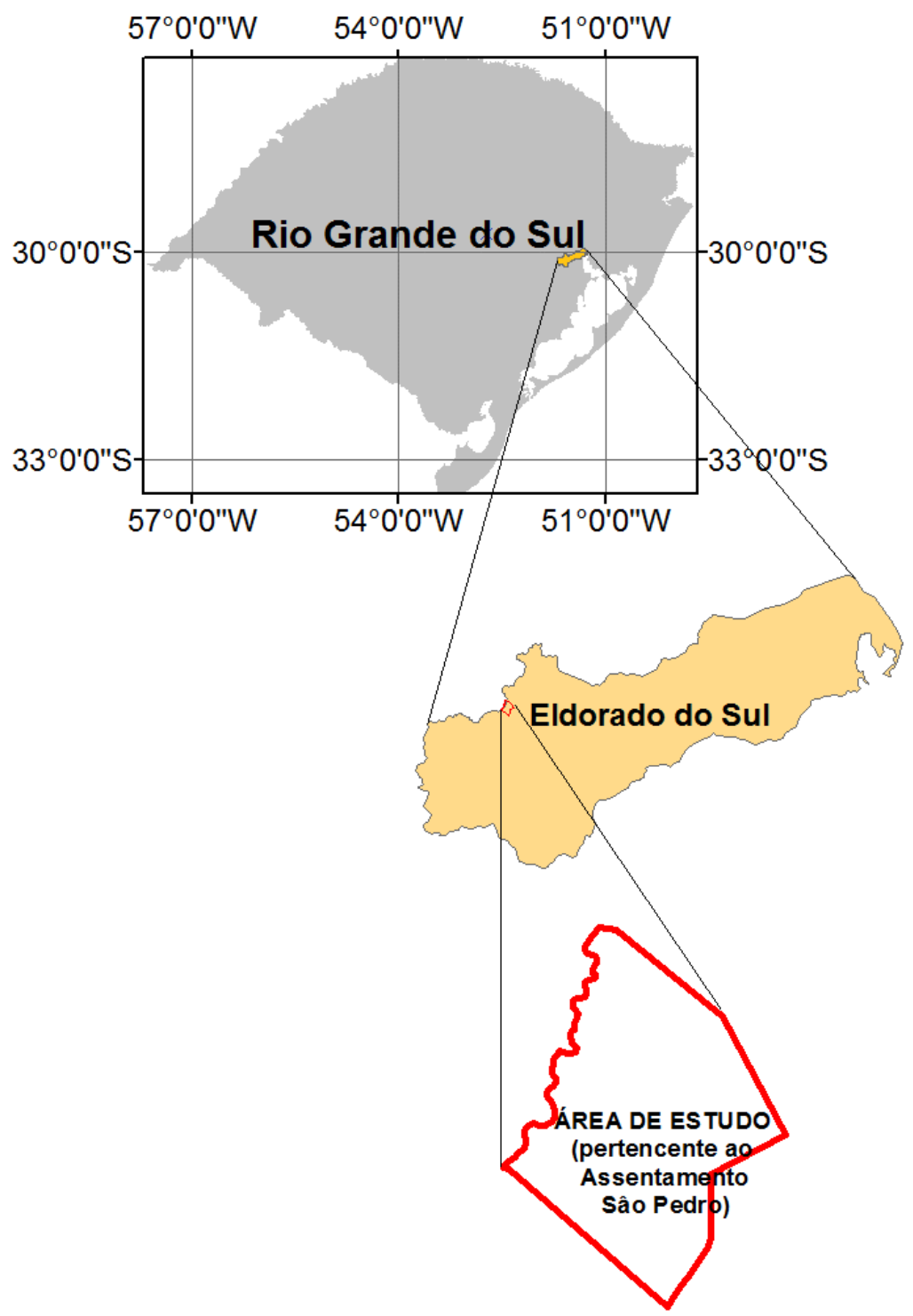

Figura 1. Localização da área de estudo (em vermelho), no município de Eldorado do Sul (em amarelo), no Estado do Rio Grande do Sul (em cinza).

Foram ocupados dois marcos da estrutura geodésica existente em área vizinha à região de estudo com os receptores configurados no método de posicionamento relativo estático. As coordenadas obtidas foram utilizadas como referência para o processamento dos demais levantamentos geodésicos, como do ponto $\mathrm{P} 01$, usado 


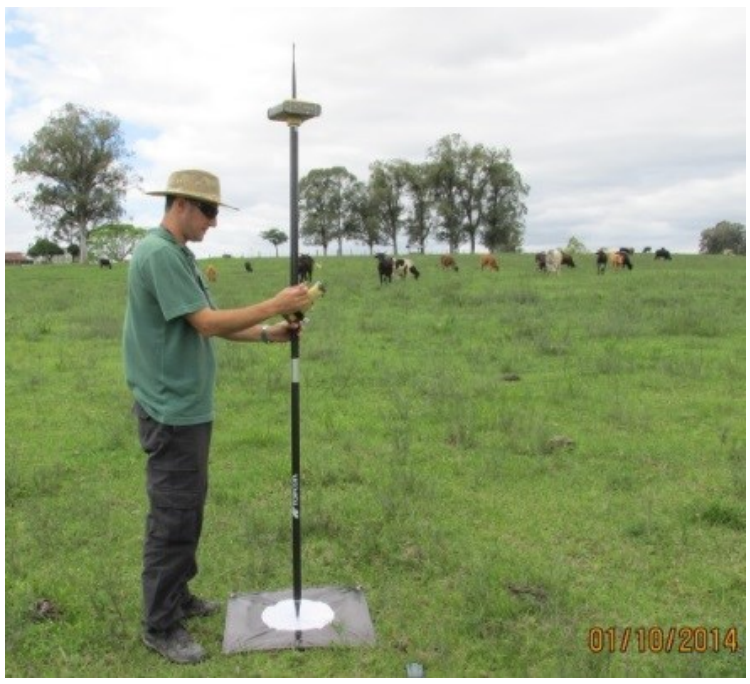

Figura 2. Levantamento de pontos de apoio ao levantamento aerofotogramétrico.

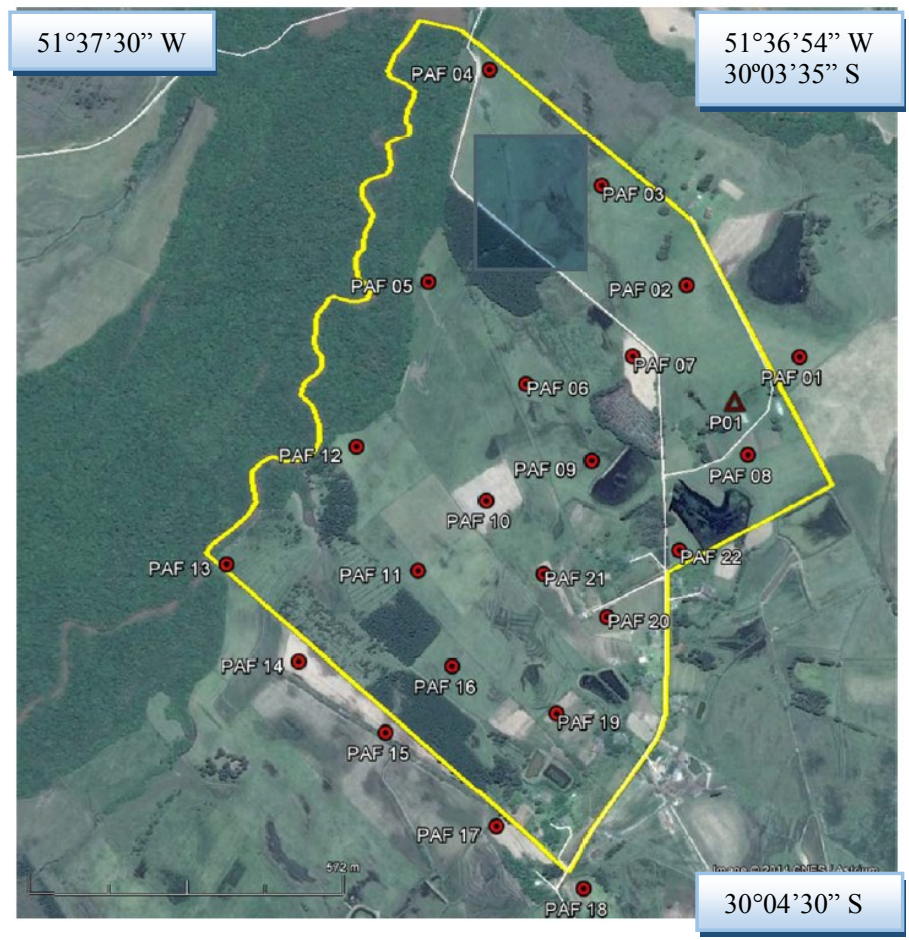

Figura 3. Distribuição de pontos de apoio fotogramétrico. 
como base para o levantamento dos pontos de apoio fotogramétrico, das feições no interior da propriedade e dos pontos de checagem para o controle de qualidade geométrica da ortofoto e da carta planimétrica, todos realizados no método de posicionamento relativo cinemático em tempo real (RTK).

Escolheu-se levantar pontos ao longo da mata ciliar, limite natural, que acompanha a margem direita do Arroio. Foram levantados, ainda, trechos de uma estrada vicinal e divisas por cerca de uma parcela do loteamento.

O referencial geodésico adotado foi o SIRGAS 2000. Utilizou-se o software TopconTools para o processamento e ajustamento das coordenadas dos marcos M08 e M09 a partir das coordenadas das estações POAL e SMAR da Rede Brasileira de Monitoramento Contínuo (RBMC). O ajustamento foi realizado de forma simultânea, considerando os três períodos de rastreio realizados. Para os levantamentos por método RTK, realizou-se um refinamento em escritório para ajustar os valores a partir de dados de estações de monitoramento contínuo.

\section{Levantamento aerofotogramétrico}

O recobrimento aerofotogramétrico abrangeu uma área aproximada de 227 ha, considerando uma margem em torno da Área de Estudo. Tendo em vista o princípio de propagação de erros à priori, característico dos processos cartográficos, trabalhou-se com uma expectativa de que a tolerância inicial fosse três vezes melhor que o erro do produto final (Rocha, 2002). Dessa forma, foi determinado que o pixel da fotografia, na escala do terreno deveria ser de $10 \mathrm{~cm}$, pois poder-seia classificar o produto na escala de referência de 1/1.000 e ainda ficaria acima da precisão requerida nas normativas do INCRA.

\section{Pontos de apoio aerofotogramétrico}

Os pontos de apoio aerofotogramétrico têm a finalidade de fornecer as coordenadas, necessárias no processo de aerotriangulação. Esses pontos foram materializados no terreno por alvos identificáveis nas fotografias (Figura 2). Como se trata de um voo apoiado, pelas especificações do equipamento, não é necessário nenhum ponto de apoio fotogramétrico. O sistema de navegação GNSS acoplado é capaz de determinar as coordenadas aproximadas do centro perspectivo da fotografia, o que facilita o processo de aerotriangulação. Contudo, para fins deste trabalho e maior controle da rigidez geométrica, definiu-se que seriam usados 22 pontos de apoio no terreno. Os alvos usados são quadrados pretos de $60 \times 60 \mathrm{~cm}$, contendo um círculo branco de $30 \mathrm{~cm}$ de diâmetro no centro. Os dados do centro geométrico de cada alvo foram obtidos pelo método do Posicionamento Relativo Cinemático, análogo ao levantamento das feições, e configurando para coleta de dez épocas em cada ponto. Dos 22 pontos de apoio planejados, foram levantados apenas 21, gravados sob a sigla PAF e numerados de 01 a 21, pois um dos alvos não foi localizado nas fotografias. A Figura 3 apresenta a distribuição dos pontos de apoio fotogramétricos na região de estudo. 


\section{Execução}

Foram executados dois voos no sentido N-S e outros dois no sentido L-O para garantir um recobrimento sem lacunas. Foi utilizado o VANT modelo Sensefly Swinglet CAM. O veículo tem peso inferior a $500 \mathrm{~g}$ e possui apenas $80 \mathrm{~cm}$ de envergadura. Possui acoplados sistema GPS, sensor de atitude (sistema inercial), transmissor de rádio, bateria para 30 minutos de voo e câmara fotográfica de 12 MP. A câmara possui distância focal nominal de $4,3 \mathrm{~mm}$, quadro focal de $4000 \mathrm{x}$ 3000 pixel e tamanho do pixel de 1,54 micrômetros e é acompanhado de software específico para programação do voo. O equipamento suporta ventos máximos de 6 $\mathrm{m} / \mathrm{s}$, ou seja, $20 \mathrm{~km} / \mathrm{h}$.

\section{Fototriangulação}

As imagens registradas e os dados de coordenadas inerciais dos respectivos voos foram armazenados e processados em um programa específico, que associa cada fotografia com seu respectivo parâmetro de orientação exterior oriundo do sistema de navegação e do sistema inercial acoplados ao VANT. Esses parâmetros são aproximados. O processamento das imagens obtidas pelo VANT é baseado na fototriangulação. O PhotoScan foi utilizado para a construção de ortofotocartas e modelos digitais de elevação georreferenciados e de alta resolução.

\section{Controle de qualidade geométrica}

A qualidade do aerolevantamento envolveu a avaliação da exatidão posicional da ortofoto e da carta planimétrica gerada pela vetorização de feições sobre a ortofoto. Comparação-se as coordenadas extraídas no produto gerado com as coordenadas homólogas levantadas no terreno. Para a coleta do conjunto de pontos para o teste, seguiu-se o critério apresentado por Rocha (2002), segundo o qual, a qualidade das coordenadas dos pontos de controle deve ser três vezes melhor que a exatidão cartográfica esperada.

\section{Qualidade da ortofoto}

A avaliação da qualidade geométrica da ortofoto serve para verificar se o produto atende aos padrões de qualidade exigidos para os documentos cartográficos, segundo critérios de exatidão pré-estabelecidos para a Cartografia Nacional. A qualidade da ortoimagem (também conhecida por ortofoto) foi avaliada mediante a verificação da exatidão posicional planimétrica, comparando-se as coordenadas de pontos extraídos de feições na ortoimagem com as coordenadas dos pontos homólogos no terreno.

De acordo com Rocha (2002), não sendo possível testar todos os pontos existentes no mapa, considera-se uma amostra que seja representativa dessa 
população, dentro de uma margem de aceitação. Para esta avaliação foi considerado um número mínimo de pontos, necessários para uma análise estatística para que fosse possível classificar o produto com um padrão de exatidão. Foram coletadas coordenadas de pontos bem definidos na imagem e de fácil localização no terreno. A amostra constituiu-se de 12 pontos que foram distribuídos na imagem de forma homogênea, ou seja, levando-se em conta a distribuição em quadrantes.

\section{Qualidade da Carta Planimétrica}

A qualidade geométrica da carta foi avaliada de forma análoga à da qualidade da ortoimagem, porém, para o cálculo do tamanho da amostra foi aplicada uma fórmula descrita por Pereira e adaptada por Rocha (2002) para uso na cartografia (Equação (4)).

$$
n=\frac{Z^{2} \gamma^{2} N}{(N-1) \varepsilon_{r}^{2}+Z^{2} \gamma^{2}}
$$

Onde:

$\mathrm{n}=$ Tamanho da amostra

$\mathrm{Z}=$ Intervalo de confiança

$\gamma=\sigma / \mu$

$\sigma=$ Desvio padrão amostral

$\mu=$ Média Amostral

$\varepsilon r=$ Erro amostral relativo

$\mathrm{N}=$ Tamanho da população

$\varepsilon=$ Erro amostral

$\varepsilon r=\varepsilon / \mu$

Para um intervalo de confiança de $90 \%$ da tabela de distribuição normal, tem-se que $Z=1,6994$. Para o cálculo de $\gamma$ foi considerado valor o desvio-padrão de três vezes menor que o valor da média. $\mathrm{O}$ valor esperado para a média amostral foi calculado pela raíz quadrada da soma dos quadrados dos erros, considerando as estimativas de erros envolvidos nas etapas de posicionamento por GNSS $(3 \mathrm{~cm})$ e o tamanho do pixel no terreno $(10 \mathrm{~cm})$, obtendo-se $\mu=10,44 \mathrm{~cm}$.

Para o cálculo do tamanho da população, levou-se em consideração o tamanho da área de recobrimento aerofotogramétrico, estimado em 200 ha. Transformandose esse valor em $\mathrm{m}^{2}$ e dividindo pelo valor quadrado do pixel no terreno $\left(10^{2}\right)$, obteve-se o valor de $\mathrm{N}=200.000 .000$. O valor do erro amostral relativo (cr) considerado foi de $12 \%$, ou 0,12 . Assim, obteve-se o tamanho da amostra de $n=21$ pontos. 
Em campo foram, inicialmente, coletadas coordenadas de 21 pontos. Analisando as diferenças entre as coordenadas Este e Norte, obtidas na carta-imagem e na vetorização, observou-se visualmente que dois pontos estariam contaminando as observações e a retirada destes valores das diferenças em $\mathrm{N}$ poderia melhorar a qualidade da vetorização. Optou-se, então, pela remoção desses pontos e ir a campo coletar mais quatro, já considerando a possibilidade de algum conter erro grosseiro e precisar ser excluído. Sendo assim, a amostra para o controle de qualidade do mapa gerado, resultou em 23 pontos.

\section{Resultados e análises}

\section{Rede Geodésica Local}

As coordenadas e respectivos desvios-padrões obtidos para os marcos M08 e M09 e para o ponto P01 são apresentados na Tabela 4. Salienta-se que POAL e SMAR são as estações de referência da RBMC, com coordenadas conhecidas, usadas para a correção diferencial das coordenadas dos marcos M08 e M09.

Verifica-se que as precisões das coordenadas dos marcos M08 e M09 enquadram-se nos padrões de precisão recomendados para o método de posicionamento relativo estático, conforme Tabela 1. Para o ponto P01, usado como base no levantamento RTK, o valor da precisão planimétrica alcançado foi de 6 $\mathrm{mm}$. $\mathrm{O}$ recomendado para linhas de base entre $5 \mathrm{e} 10 \mathrm{~km}$ é de 5 à $10 \mathrm{~mm}+/-1 \mathrm{ppm}$, portanto, a precisão das coordenadas apresenta valores melhores que os parâmetros recomendados.

\section{Tabela 4}

Resumo das coordenadas e precisões dos marcos e do ponto de apoio geodésico

\begin{tabular}{lcccccccc}
\hline & \multicolumn{3}{c}{ Coordenadas UTM } & \multicolumn{5}{c}{ Desvios padrões } \\
\cline { 2 - 8 } Nome & Norte $(m)$ & Este $(m)$ & Altitude $(m)$ & $N(m)$ & $E(m)$ & $H z(m)$ & $h(m)$ \\
\hline POAL & 6673004.053 & 488457.545 & 76.750 & 0.001 & 0.002 & 0.002 & 0.002 \\
SMAR & 6709269.527 & 237205.247 & 113.110 & 0.001 & 0.001 & 0.001 & 0.001 \\
M08 & 6670229.928 & 434741.180 & 53.899 & 0.006 & 0.006 & 0.009 & 0.014 \\
M09 & 6670607.177 & 434886.626 & 55.563 & 0.006 & 0.007 & 0.009 & 0.014 \\
P01 & 6673567.621 & 440718.147 & 28.176 & 0.004 & 0.005 & 0.006 & 0.011 \\
\hline
\end{tabular}

Fonte: Elaboração própria. 


\section{Pontos de apoio aerofotogramétrico}

As coordenadas desses pontos, bem como as precisões obtidas estão apresentadas na Tabela 5. Observa-se que o maior valor do desvio-padrão planimétrico foi de 0,012 m, referente ao PAF_21, portanto, a qualidade dos pontos de apoio apresentou resultados melhores que a tolerância estabelecida, que é de $0,3 \mathrm{~m}$ para levantamentos por GNSS.

Tabela 5

Coordenadas e precisões dos pontos de apoio fotogramétrico

\begin{tabular}{cccccccc}
\hline & & & & & StdDev & StdDev & StdDev \\
me & Norte $(m)$ & Este $(m)$ & Altitude $(m)$ & $n(m)$ & $e(m)$ & $H z(m)$ & $u(m)$ \\
\hline P01 & 6673567.621 & 440718.147 & 28.176 & 0.004 & 0.005 & 0.006 & 0.011 \\
PAF_01 & 6673651.337 & 440836.585 & 24.963 & 0.006 & 0.007 & 0.010 & 0.015 \\
PAF_02 & 6673778.705 & 440631.112 & 26.344 & 0.006 & 0.007 & 0.009 & 0.015 \\
PAF_03 & 6673958.331 & 440475.280 & 19.458 & 0.006 & 0.007 & 0.010 & 0.015 \\
PAF_04 & 6674168.577 & 440269.992 & 17.524 & 0.005 & 0.006 & 0.008 & 0.013 \\
PAF_05 & 6673783.585 & 440163.343 & 18.248 & 0.005 & 0.005 & 0.007 & 0.012 \\
PAF_06 & 6673600.221 & 440339.576 & 18.819 & 0.005 & 0.005 & 0.007 & 0.012 \\
PAF_07 & 6673651.041 & 440533.869 & 24.277 & 0.005 & 0.005 & 0.007 & 0.012 \\
PAF_08 & 6673472.330 & 440743.895 & 26.940 & 0.005 & 0.006 & 0.007 & 0.012 \\
PAF_09 & 6673460.293 & 440460.215 & 20.121 & 0.005 & 0.005 & 0.007 & 0.013 \\
PAF_10 & 6673387.320 & 440268.805 & 20.032 & 0.006 & 0.007 & 0.009 & 0.018 \\
PAF_11 & 6673258.420 & 440147.645 & 19.981 & 0.007 & 0.008 & 0.011 & 0.022 \\
PAF_12 & 6673483.391 & 440033.857 & 18.119 & 0.005 & 0.006 & 0.008 & 0.015 \\
PAF_13 & 6673268.129 & 439798.768 & 18.245 & 0.006 & 0.006 & 0.009 & 0.015 \\
PAF_14 & 6673093.831 & 439932.103 & 20.738 & 0.006 & 0.006 & 0.008 & 0.014 \\
PAF_15 & 6672966.701 & 440090.375 & 20.889 & 0.005 & 0.006 & 0.008 & 0.014 \\
PAF_16 & 6673087.047 & 440210.718 & 20.673 & 0.006 & 0.006 & 0.008 & 0.014 \\
PAF_17 & 6672798.140 & 440291.074 & 23.507 & 0.005 & 0.006 & 0.008 & 0.019 \\
PAF_18 & 6672690.670 & 440450.452 & 27.363 & 0.005 & 0.006 & 0.008 & 0.014 \\
PAF_19 & 6673003.399 & 440399.093 & 24.314 & 0.006 & 0.006 & 0.008 & 0.014 \\
PAF_20 & 6673178.060 & 440490.309 & 27.269 & 0.005 & 0.006 & 0.008 & 0.014 \\
PAF_21 & 6673253.995 & 440373.845 & 22.324 & 0.010 & 0.008 & 0.012 & 0.020 \\
PAF_22 & 6673298.260 & 440620.428 & 25.490 & 0.006 & 0.006 & 0.008 & 0.013 \\
\hline
\end{tabular}

Fonte: Elaboração própria. 


\section{Controle de qualidade geométrica}

Avaliou-se a qualidade geométrica da carta planimétrica — produto final-e optouse também por analisar a qualidade geométrica da ortofoto. Foram levantadas coordenadas de feições bem definidas na carta e na ortofoto e comparadas com as coordenadas das feições homólogas no terreno, levantadas por GNSS. Utilizou-se o método descrito por Galo e Camargo (1994), baseados em Merchant (1982), para complementar a legislação e analisar a consistência dos dados.

\section{Qualidade geométrica da ortofoto}

Para o controle de qualidade geométrica da ortofoto, foram considerados 12 pontos. O resultado das discrepâncias entre as coordenadas dos pontos checagem, extraídos do ortofoto e obtidos por GNSS, encontra-se na Tabela 6. Observa-se, que $90 \%$ dos pontos bem definidos, quando comparados, apresentam erro RMS (erro médio quadrático) inferior a $0,279 \mathrm{~m}$. A Figura 4 apresenta a ortofotomosaico desenvolvida.

Tabela 6

Diferenças entre coordenadas dos pontos de checagem da ortofotomosaico e coordenadas GNSS

\begin{tabular}{|c|c|c|c|c|c|c|c|}
\hline \multirow[b]{2}{*}{ Pto } & \multicolumn{2}{|c|}{$\begin{array}{c}\text { Coordenadas } \\
\text { ortofotomosaico }\end{array}$} & \multicolumn{2}{|c|}{ Coordenadas GNSS } & \multirow[b]{2}{*}{$\Delta E(m)$} & \multirow[b]{2}{*}{$\Delta N(m)$} & \multirow[b]{2}{*}{$R M S(m)$} \\
\hline & $E(m)$ & $N(m)$ & $E(m)$ & $N(m)$ & & & \\
\hline 1 & 440593,263 & 6673678,88 & 440593,128 & 6673678,903 & $-0,134514$ & 0,02377 & 0,1365980 \\
\hline 2 & 440428,479 & 6674047,37 & 440428,241 & 6674047,212 & $-0,238325$ & $-0,15921$ & 0,28661233 \\
\hline 3 & 440762,621 & 6673472,67 & 440762,523 & 6673472,730 & $-0,097627$ & 0,06265 & 0,11600023 \\
\hline 4 & 440643,942 & 6673885,71 & 440643,715 & 6673885,655 & $-0,226535$ & $-0,05267$ & 0,2325773 \\
\hline 5 & 440175,745 & 6673539,11 & 440175,713 & 6673539,101 & $-0,031515$ & $-0,00645$ & 0,03216827 \\
\hline 6 & 439967,895 & 6673378,93 & 439968,174 & 6673378,948 & 0,279071 & 0,01698 & 0,2795870 \\
\hline 7 & 440154,608 & 6673224,11 & 440154,608 & 6673224,144 & $-0,000399$ & 0,03312 & 0,0331224 \\
\hline 8 & 440224,06 & 6673079,99 & 440224,047 & 6673079,874 & $-0,012899$ & $-0,11143$ & 0,1121741 \\
\hline 9 & 440359,762 & 6673572,14 & 440359,737 & 6673572,148 & $-0,025316$ & 0,00347 & 0,0255527 \\
\hline 10 & 440396,752 & 6672744,60 & 440396,598 & 6672744,671 & $-0,154022$ & 0,07335 & 0,1705960 \\
\hline 11 & 440568,838 & 6673121,71 & 440568,755 & 6673121,677 & $-0,082719$ & $-0,02859$ & 0,0875204 \\
\hline 12 & 440211,250 & 6673960,66 & 440210,982 & 6673960,646 & $-0,267718$ & $-0,01063$ & 0,2679289 \\
\hline \multicolumn{7}{|c|}{ Média RMS } & 0,14836983 \\
\hline \multicolumn{7}{|c|}{ Desvio padrão RMS } & 0,0982928 \\
\hline \multicolumn{7}{|c|}{ Qualidade pela soma de três desvios padrões e a média dos erros médios quadráticos } & 0,44324823 \\
\hline
\end{tabular}

Fonte: Elaboração própria. 


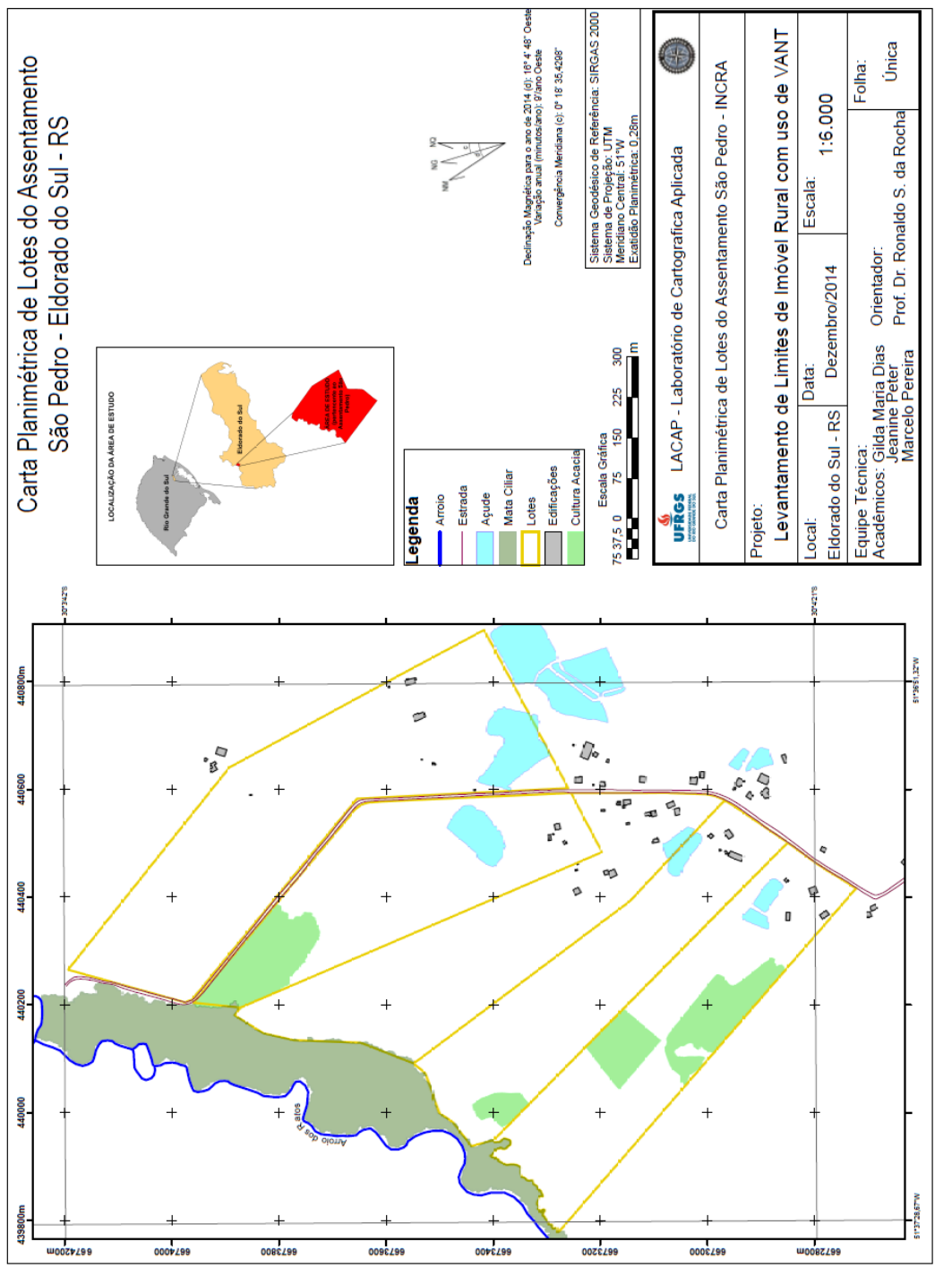

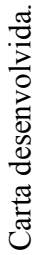

承 


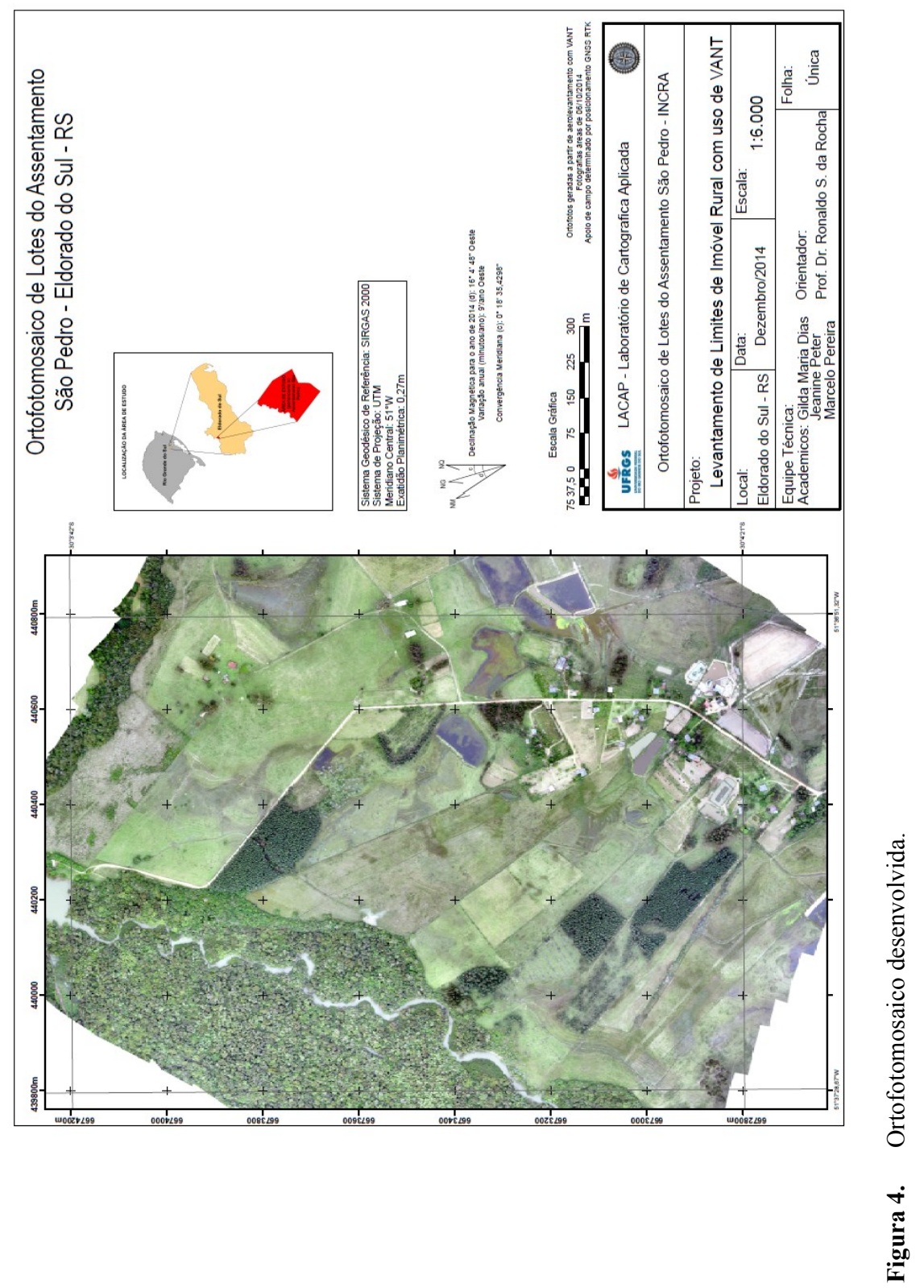


$\mathrm{Na}$ análise de tendência (Tabela 7), verificou-se que, em módulo, o valor de $t$ calculado para a coordenada $E$ apresenta tendência, uma vez que o valor é superior ao do $t$ tabelado, que é de 1,7959 , para $n=12(n-1=11)$ e nível de significância de $10 \%$ (considerando bicaudal, tem-se 0,05 para cada lado). Já na coordenada $\mathrm{N}$, o valor em módulo é inferior ao t tabelado, ou seja, não há tendência.

As Tabelas 8 e 9 apresentam os resultados das análises de tendência e de precisão, respectivamente. A ortofoto apresentou tendência na componente Este (E). Desta forma tem-se que desenvolver uma análise mais profunda. Provavelmente esta tendência pode ter sido causada por um erro sistemático, ou pelo reduzido tamanho da amostra.

\section{Qualidade geométrica da carta planimétrica}

Foram testados 23 pontos para a avaliação da qualidade geométrica da carta. As coordenadas desses pontos foram extraídas da carta elaborada, localizados em cantos de edificações e moirões de cercas. O resultado das discrepâncias entre as coordenadas dos pontos de checagem é apresentado na Tabela 10. Observa-se que todos os pontos apresentam erro RMS abaixo dos 0,50 m exigidos pelo INCRA para precisão em limites artificiais. Pode-se constatar, ainda, que 90\% dos pontos bem definidos na carta, quando testados no terreno, possuem exatidões melhores que 0,282 m. A Tabela 11 apresenta os dados obtidos.

\section{Tabela 7}

Estatísticas descritivas da ortoimagem

\begin{tabular}{lrr}
\hline \multicolumn{1}{c}{ Componente } & \multicolumn{1}{c}{$\Delta E$} & \multicolumn{1}{c}{$\Delta N$} \\
\hline Número de Pontos & 12 & 12 \\
Média (m) & $-0,08270983$ & $-0,01297$ \\
Variância $\left(\mathrm{m}^{2}\right)$ & 0,021415487 & 0,004614499 \\
Desvio-Padrão (m) & 0,146340313 & 0,067930103 \\
Mínimo (m) & $-0,267718$ & $-0,15921$ \\
Mediana (m) & $-0,090173$ & $-0,00149$ \\
Máximo (m) & 0,279071 & 0,07335 \\
\hline
\end{tabular}

Fonte: Elaboração própria.

Tabela 8

Análise de tendência na ortoimagem

\begin{tabular}{ccc}
\hline Coordenada & $E$ & $N$ \\
\hline $\mathrm{t}_{\text {calc }}$ & $-1,95786972$ & $-0,66140629$ \\
$\mathrm{t}_{(12 ; 5 \%)}$ & 1,7959 & 1,7959 \\
Possui tendência? & Sim & Não \\
\hline
\end{tabular}


Tabela 9

Análise de precisão da ortofoto

\begin{tabular}{|c|c|c|}
\hline Coordenada & $E$ & $N$ \\
\hline$\sigma_{\mathrm{A}}^{2}$ & \multicolumn{2}{|c|}{0,01445} \\
\hline$\chi_{\text {cálculado }}^{2}$ & 16,30244701 & 3,512767355 \\
\hline$\chi_{(23,10 \%)}^{2}$ & 17,275 & 17,275 \\
\hline Classe & A & A \\
\hline
\end{tabular}

Fonte: Elaboração própria.

Tabela 10

Diferenças entre as coordenadas da carta e as levantadas por GNSS

\begin{tabular}{lllllllll}
\hline \multicolumn{7}{c}{ Coordenadas } & Ortofotocarta & \multicolumn{2}{c}{ Coordenadas $G N S S$} \\
$N^{o}$ & $E(m)$ & $N(m)$ & $E(m)$ & \multicolumn{1}{c}{$N(m)$} & \multicolumn{1}{c}{$\Delta E(m)$} & $\Delta N(m)$ & Erro RMS \\
\hline 2 & 440640,1627 & 6673892,295 & 440639,956 & 6673892,473 & $-0,206716$ & 0,17786 & 0,27270 \\
3 & 440810,3674 & 6673576,612 & 440810,341 & 6673576,692 & $-0,026446$ & 0,07993 & 0,08419 \\
4 & 440895,3838 & 6673416,764 & 440895,611 & 6673416,804 & 0,227163 & 0,04014 & 0,23068 \\
5 & 440792,9952 & 6673361,305 & 440793,076 & 6673361,501 & 0,080826 & 0,19568 & 0,21171 \\
6 & 440582,3977 & 6673653,404 & 440582,425 & 6673653,124 & 0,027336 & 0,28047 & 0,28179 \\
7 & 440787,7064 & 6673595,53 & 440787,601 & 6673595,463 & $-0,10544$ & 0,06748 & 0,12518 \\
8 & 440592,8513 & 6673254,257 & 440592,837 & 6673254,24 & $-0,014328$ & 0,01696 & 0,02220 \\
9 & 440606,6297 & 6673211,357 & 440606,492 & 6673211,413 & $-0,137731$ & 0,05566 & 0,14855 \\
10 & 440558,7465 & 6673197,346 & 440558,608 & 6673197,392 & $-0,138479$ & 0,04623 & 0,14599 \\
11 & 440449,5005 & 6673241,502 & 440449,517 & 6673241,506 & 0,016536 & 0,00361 & 0,01692 \\
12 & 440391,7933 & 6673144,809 & 440391,768 & 6673144,952 & $-0,025279$ & 0,14274 & 0,14496 \\
14 & 440500,8328 & 6672849,765 & 440500,969 & 6672849,74 & 0,13625 & 0,02498 & 0,13852 \\
15 & 439786,066 & 6673270,515 & 439786,193 & 6673270,494 & 0,127033 & 0,02097 & 0,12875 \\
16 & 440397,5677 & 6673396,209 & 440397,506 & 6673396,108 & $-0,06172$ & $-0,1014$ & 0,11870 \\
17 & 440429,9293 & 6672846,737 & 440429,692 & 6672846,737 & $-0,237328$ & $1,0001 \mathrm{E}$ & 0,23732 \\
18 & 440265,3407 & 6673314,22 & 440265,459 & 6673314,533 & 0,118287 & 0,31307 & 0,33467 \\
19 & 440572,7178 & 6673076,03 & 440572,67 & 6673076,35 & $-0,04783$ & 0,31999 & 0,32354 \\
20 & 440499,6477 & 6673102,44 & 440499,576 & 6673102,436 & $-0,071705$ & 0,00354 & 0,07179 \\
21 & 440513,0132 & 6672997,528 & 440512,993 & 6672997,534 & $-0,020159$ & 0,00602 & 0,02103 \\
22 & 440656,1166 & 6673532,9 & 440656,074 & 6673532,976 & $-0,042648$ & 0,07627 & 0,08738 \\
23 & 440792,4524 & 6673562,18 & 440792,363 & 6673562,224 & $-0,089413$ & 0,04431 & 0,09979 \\
25 & 440486,5907 & 6672789,052 & 440486,513 & 6672789,016 & $-0,077679$ & 0,03595 & 0,08559 \\
26 & 440265,0639 & 6674193,302 & 440265,182 & 6674193,067 & 0,118062 & 0,23462 & 0,26265 \\
\hline Média RMS & & & & & & 0,13825 \\
Desvio Padrão RMS & & & & & & 0,10298 \\
Qualidade dada pela soma de 3 desvios padrões e a média dos erros médios quadráticos & 0,44720 \\
\hline
\end{tabular}

Fonte: Elaboração própria. 
Tabela 11

Estatísticas descritivas $(\Delta \mathrm{E}$ e $\Delta \mathrm{N})$ da carta

\begin{tabular}{lrr}
\hline \multicolumn{1}{c}{ Componente } & \multicolumn{1}{c}{$\Delta E$} & \multicolumn{1}{c}{$\Delta N$} \\
\hline Número de Pontos & 23 & 23 \\
Média (m) & $-0,019626435$ & 0,031092609 \\
Variância $\left(\mathrm{m}^{2}\right)$ & 0,013194782 & 0,020033525 \\
Desvio-Padrão (m) & 0,114868543 & 0,141539835 \\
Mínimo (m) & $-0,237328$ & $-0,280470001$ \\
Mediana (m) & $-0,026446$ & 0,00602 \\
Máximo (m) & 0,227163 & 0,31999 \\
\hline
\end{tabular}

Fonte: Elaboração própria.

Tabela 12

Análise estatística de tendência na carta

\begin{tabular}{lcc}
\hline \multicolumn{1}{c}{ Coordenada } & $E$ & $N$ \\
\hline $\mathrm{t}_{\text {calc }}$ & $-0,819415589$ & 1,053519055 \\
$\mathrm{t}_{(23,5 \%)}$ & 1,7171 & 1,7171 \\
Possui tendência? & Não & Não \\
\hline
\end{tabular}

Fonte: Elaboração própria.

Tabela 13

Análise de precisão da carta planimétrica

\begin{tabular}{lcc}
\hline \multicolumn{1}{c}{ Coordenada } & $E$ & $N$ \\
\hline$\sigma^{2} \mathrm{~A}$ & 0,01445 & 0,01445 \\
$\chi^{2}$ cálculado & 20,08894161 & 30,50086818 \\
$\chi^{2}(23 ; 10 \%)$ & 30,813 & 30,813 \\
Classe & $\mathrm{A}$ & $\mathrm{A}$ \\
\hline
\end{tabular}

Fonte: Elaboração própria.

Pelo resultado do teste $\mathrm{t}$, verifica-se que a exatidão planimétrica não apresenta tendência em nenhuma das componentes $(E$ e $N)$, conforme Tabela 12. Quanto à precisão (Tabela 13), verifica-se, pelo teste baseado na distribuição Qui-quadrado, que o produto pode ser classificado no PEC-PCD como sendo da Classe A para a escala 1:1.000. A Figura 5 apresenta a carta gerada. 


\section{Conclusão}

Através do controle de qualidade geométrica, observou-se uma de exatidão de 0,279 m para $90 \%$ das coordenadas extraídas da ortofoto e de 0,282 m para as coordenadas resultantes da carta planimétrica. Esse resultado se mostrou coerente, uma vez que a qualidade da ortofoto é melhor do que a da base cartográfica gerada (carta). Por outro lado, observou-se que houve tendência na componente Este (E) das coordenadas da ortofoto, fato que não se detectou nos testes com a carta. Este resultado suscitou uma aparente contradição, tendo em vista que uma tendência deveria ser também evidenciada no teste da carta. Uma possibilidade para este resultado pode estar no menor número de pontos para a checagem da qualidade da ortofoto (12). Outra possibilidade pode ser devido à distribuição não homogênea dos pontos de controle de qualidade da carta.

A partir dos objetivos propostos e dos resultados obtidos, conclui-se que a técnica usada é compatível com os requisitos e precisões exigidos pelas normativas vigentes. A técnica mostrou-se uma alternativa viável para levantamento em limites naturais, onde as precisões podem ser de $3,0 \mathrm{~m}$ ou de até $7,50 \mathrm{~m}$ em regiões inacessíveis, conforme preconiza o Manual Técnico de Limites e Confrontações. Embora o referido manual não permita o uso desse método em vértices de limites secos, verificou-se que as precisões requeridas para essa categoria de vértice, $50 \mathrm{~cm}$ ou melhores, também podem ser alcançadas com o uso do método.

$\mathrm{O}$ uso de VANT, por ser um equipamento que apresenta forte crescimento no uso atualmente, carece de normas de segurança e utilização para diferentes fins. Esse método pode ser uma alternativa quando a disponibilidade de tempo e de recursos é limitada.Melhores resultados podem ser obtidos com voos programados para maior resolução espacial no terreno. Deve-se considerar também o custobenefício do método, uma vez que a quantidade de fotografias aumenta significativamente com o aumento da resolução espacial e, consequentemente, o processo fotogramétrico se torna mais oneroso e exige maior capacidade computacional para armazenamento e processamento. Estudos mais detalhados podem ser observados em Ruy (2008).

Por fim, alerta-se que o uso do VANT para obter fotografías aéreas para mapeamento deve ser utilizado com critério, seguindo todos os preceitos científicos e técnicos da cartografia topográfica.

\section{Bibliografia}

Andrade, J.B. (1998). Fotogrametria, SBEE, Curitiba.

Brasil (1984). "Decreto $\mathrm{n}^{\circ}$ 89.817, de 20 de junho de 1984". Estabelece as Instruções Reguladoras das Normas Técnicas da Cartografia Nacional, disponível em: <http://www.planalto.gov.br/ccivil_03/decreto/19801989/D89817.htm>, acesso em: 03 nov. 2014. 
, (2001). "Lei no 10.267, de 28 de agosto de 2001". Altera dispositivos das Leis $\mathrm{n}^{\circ}$ 4.947, de 6 de abril de 1966, 5.868, de 12 de dezembro de 1972, 6.015, de 31 de dezembro de 1973, 6.739, de 5 de dezembro de 1979, 9.393, de 19 de dezembro de 1996, e dá outras providências, disponível em $<$ http://www.planalto.gov.br/ccivil_03/leis/leis_2001/110267.htm>, acesso em: 03 nov. 2014.

Comissão Nacional de Cartografia — CONCAR (2011). Padrão de Exatidão Cartográfica Digital. Exército Brasileiro. Brasil.

Galo, M. e Camargo, P. (1994). "O uso do GPS no controle de qualidade de cartas", COBRAC-1994, $1^{\circ}$ Congresso Brasileiro de Cadastro Técnico Multifinalitário, tomo II, Florianópolis, p. 41-48.

Instituto Brasileiro de Geografia e Estatística IBGE. (2008). Recomendações para levantamentos relativos estáticos - GPS, disponível em $<\mathrm{ftp}$ //geoftp.ibge.gov.br/documentos/geodesia/pdf/recom_gps_internet.pdf $>$, acesso em: 12 nov. 2014.

— 2014. "Cidades", disponível em: $<$ http://www.cidades.ibge.gov.br $>$, acesso em: 25 nov. 2014.

Instituto Nacional de Colonização e Reforma Agrária - INCRA (2013). Norma Técnica para Georreferenciamento de Imóveis Rurais, 3a. ed., Coordenação Geral de Cartografia, Brasília, disponível em <http://www.incra.gov. br/media/institucional/norma técnica para georreferenciamento de imóveis rurais 3 ediçao.pdf $>$, acesso em: 06 set. 2014.

— 2013. Manual Técnico de Posicionamento, 1a. ed., Coordenação Geral de Cartografia, Brasília, disponível em <http://www.incra.gov.br/media/ institucional/manual técnico de posicionamento 1 ediçao.pdf $>$, acesso em: 06 set. 2014.

, 2013. Manual Técnico de Limites e Confrontações, 1a. ed., Coordenação

Geral de Cartografia, Brasília, disponível em <http://www.incra. gov.br/media/institucional/manual técnico de limites e confrontaçoes 1 edicao.pdf $>$, acesso em: 06 set. 2014.

Lima, D.L. et al. (2014). Restituição e Geração de Ortoimagem a partir de um Estereopar EROS", UNESP, disponível em <https://www.ufpe. $\mathrm{br} / \mathrm{cgtg} / \mathrm{ISIMGEO} / \mathrm{CD} / \mathrm{html} /$ Fotogrametria e Sensoriamento Remoto/Artigos/ f038.pdf>, acesso em: 30 nov. 2014.

Oliveira, L.F. "Análise de Modelos Digitais de Superfície gerados a partir de imagens do sensor PRISM/ALOS", 184 f. Dissertação (Mestrado). Viçosa, MG, Universidade Federal de Viçosa (UFV), 2011.

Prado, A. e Krueger, C.P. (2003). “Análise da Acurácia nos Posicionamentos Diferenciais aplicando as técnicas DGPS e RTK”, Revista Brasileira de 
Cartografia, 1, (55), 29-38, disponível em <http://www.lsie.unb.br/rbc/ index.php/rbc/article/view/179>, acesso em: 20 nov. 2014.

Rocha, R. dos S. (2002). "Exatidão Cartográfica para as cartas digitais urbanas", Tese (Doutorado), Programa de Pós Graduação em Engenharia de Produção, (UFSC), Florianópolis, disponível em: <http://www.ufrgs.br/engcart/tese RochaRS.pdf>, acesso em: $01 \mathrm{dez} .2014$.

Ruy, R.S. (2008). "Desenvolvimento e validação geométrica de um sistema para mapeamento com câmaras digitais de médio formato", Tese (Doutorado), Programa de Pós Graduação em Ciências Cartográficas, UNESP, Presidente Prudente [s/n], 2008. 